\title{
Fiabilidad de la cinemática angular de rodilla y tobillo de la carrera en el plano sagital medido con Younext Direct Motion Capture ${ }^{\circledR}$
}

\author{
Andrés Ráfales Perucha ${ }^{1}$; Antonio Gómez Bernal2; Javier Alfaro Santafé3; Alejandro-Jesús Almenar \\ Arasanz $^{4}$
}

Fecha de recepción: 11 de septiembre de 2018 / Fecha de aceptación: 2 de enero 2019.

Resumen. En los últimos cuarenta años la popularidad de salir a correr o hacer running se ha incrementado notablemente. Su popularidad y el gran número de lesiones hace que el running sea la actividad física más estudiada por el área de la biomecánica. En la práctica clínica, se utilizan principalmente software de análisis de movimiento 2D para analizar la cinemática. Younext Direct Motion Capture ${ }^{\circledR}$ (Podoactiva S.L., Huesca) es un software que analiza los parámetros cinemáticos durante la carrera en el plano sagital. El objetivo general de este estudio fue determinar la fiabilidad y repetibilidad de la cinemática angular de la rodilla y del tobillo de la carrera en el plano sagital en población sana utilizando Younext Direct Motion Capture ${ }^{\circledR}$. Estudio piloto observacional de concordancia. 8 sujetos (5 varones y 3 mujeres) fueron filmados mientras corrían en una cinta de correr, obteniendo tres grabaciones del ciclo de la carrera de cada participante en la primera sesión. Se realizó una segunda sesión de grabaciones a los 7 días del primer registro para volver a grabar siguiendo el mismo protocolo. Se calculó el coeficiente de correlación intraclase (ICC) para determinar la fiabilidad intra-sesión e inter-sesión del software a la hora de medir la cinemática de la rodilla y del tobillo. Se obtuvo una fiabilidad intra-sesión e inter-sesión casi perfectas (ICC $>0,81$ ) tanto para tobillo como para rodilla. Este estudio demostró que el software Younext Direct Motion Capture ${ }^{\circledR}$ es fiable para analizar la cinemática angular de la rodilla y del tobillo de la carrera en población adulta sana.

Palabras clave: fiabilidad; biomecánica; software de análisis del movimiento; cinemática; carrera.

\section{[en] Reliability of the angular kinematic of knee and ankle during the running in the sagittal plane measured with Younext Direct Motion Capture ${ }^{\circledR}$}

Abstract. The popularity of running has increased remarkably the past forty years. Its popularity and the large number of injuries make running the most studied physical activity in the area of biomechanics. In clinical practice, joint kinematics during running are primarily quantified by two-dimensional motion-analysis software. Younext Direct Motion Capture $^{\circledR}$ (Podoactiva S.L., Huesca) is a software that analyzes the kinematic parameters during the running in the sagittal plane. The main goal of this study was to determine the reliability and repeatability of the angular kinematics of the knee and ankle in the sagittal plane in a healthy population using Younext Direct Motion Capture ${ }^{\mathbb{R}}$. Observational pilot study of concordance. 8 subjects ( 5 men and 3 women) were filmed while running on a treadmill, obtaining three recordings of the running cycle of each participant in the first session. A second session of recordings was made 7 days after the first recording to re-record following the same protocol. The intraclass correlation coefficient (ICC) was calculated to determine the intra-session reliability and inter-session reliability of the software when measuring the kinematics of the knee and the ankle. Very good intra-session and inter-session reliability (ICC $>0.81$ ) were obtained for both ankle and knee measurements. This study showed that Younext Direct Motion Capture ${ }^{\circledR}$ software is reliable for analyzing the angular kinematics of the knee and ankle during running in a healthy adult population.

\footnotetext{
Graduado en Fisioterapia. Máster Oficial en Investigación en Ciencias de la Salud (USJ) arafales@usj.es

2 Diplomado en Podología. Máster Oficial investigación en Podología (URJC). Doctor por la Universidad Rey Juan Carlos. Profesor del Máster oficial de Investigación en Ciencias de la Salud (USJ)

Edificio Podoactiva. Parque Tecnológico Walqa, Ctra. N330a Km 566, 22197. Cuarte, Huesca. antoniogomez@podoactiva.com

3 Diplomado en Podología. Profesor del Máster oficial de Investigación en Ciencias de la Salud (USJ). Máster Oficial en Gerontología Social (UNIZAR). Doctor por la Universidad Europea javieralfaro@podoactiva.com

4 Graduado en Ciencias de la Actividad Física y el Deporte aj.almenar@gmail.com
} 
Keywords: reliability; biomechanics; motion-analysis software; kinematics; running.

Sumario: 1. Introducción. 2. Material y métodos. 3. Resultados. 4. Discusión. 5. Bibliografía.

Cómo citar: Ráfales Perucha, A. et ali (2019) Fiabilidad de la cinemática angular de rodilla y tobillo de la carrera en el plano sagital medido con Younext Direct Motion Capture ${ }^{\circledR}$, en Revista internacional de ciencias podológicas 13(1), 47-54.

Los autores declaran no tener ningún tipo de interés económico o comercial.

\section{Introducción}

Cada vez hay más evidencia sobre la actividad física regular, como caminar o correr, y sus beneficios para la salud(1). La actividad física regular influye positivamente en la prevención primaria y secundaria de enfermedades cardiovasculares, cáncer, hipertensión, diabetes y otras enfermedades crónicas(2) e incrementa la calidad de vida(3). Varias revisiones y metanálisis han demostrado la asociación positiva entre el caminar y la prevención de eventos de enfermedad cardiovascular en la población general(4-6).

En los últimos cuarenta años la popularidad de salir a correr o hacer running se ha incrementado notablemente(7). Desafortunadamente, las lesiones relacionadas con este deporte han sido la principal causa de una retirada permanente en la práctica de esta actividad física(8), con una incidencia acumulada anual de lesiones superior al $85 \%$ en población general corredora(9)duration, frequency, and intensity. Su popularidad y el gran número de lesiones hace que el running sea la actividad física más estudiada por el área de la biomecánica.

Los métodos que se han utilizado tradicionalmente para el análisis cinemático de la marcha y de la carrera en el ámbito clínico, como la observación visual por sí sola o en combinación con las grabaciones de cronómetro(10), han desaparecido ya que presentan una baja fiabilidad inter-observador. En el área de la biomecánica existen sofisticados instrumentos de medición que proporcionan datos válidos y fiables para el análisis de la marcha y la carrera tales como los sistemas de análisis de cinemática tridimensionales(11), plataformas de presiones(12), plantillas instrumentadas(13), acelerómetros(14)...
Las principales desventajas de estos sistemas son su alto coste, la fragilidad del sensor y la complejidad de funcionamiento, por lo que se usan principalmente para la investigación y no para fines clínicos. Sin embargo, los sistemas de análisis cinemáticos bidimensionales son más económicos y fáciles de utilizar, por lo que su uso en el ámbito clínico para analizar la cinemática es más frecuente(15-20). Se ha demostradoquemedianteun análisis cinemático de la carrera únicamente en el plano sagital se puede obtener información importante sobre la cinemática de la carrera(21), ya que los principales movimientos del cuerpo durante la marcha y la carrera se realizan en este plano. Además, se han observado relaciones importantes entre el análisis $2 \mathrm{D}$ y $3 \mathrm{D}$ en todas las articulaciones en el plano sagital(22). A pesar de la falta de precisión y la incapacidad de capturar las rotaciones, los sistemas de análisis cinemático bidimensionales pueden proporcionar un método práctico para evaluar el desplazamiento de la articulación en el plano sagital y valorar el riesgo de lesión en la extremidad inferior(22).

Younext Direct Motion Capture ${ }^{\circledR}$ (Podoactiva S.L., Huesca) es un software que analiza los parámetros cinemáticos durante la carrera en el plano sagital. A diferencia de otros sistemas de análisis informatizados, este sistema cuenta con un rastreo o tracking automático que facilita la valoración de los pacientes. El tracking automático de este software está basado en la función informática OpenCV tracking $R G B$ que consiste en fijar el seguimiento de un pixel asociado a un determinado color a lo largo de una filmación, obviando el resto de colores(23).

El objetivo general de este estudio es determinar la fiabilidad y repetibilidad de la cinemática angular de la rodilla y del tobillo de 
la carrera en el plano sagital en población sana utilizando Younext Direct Motion Capture ${ }^{\circledR}$.

\section{Material y métodos}

Durante el mes de julio de 2018 se realizó un estudio piloto observacional de concordancia. El estudio siguió los principios éticos de la Declaración de Helsinki y fue aprobado por el Comité Ético de la Comunidad de Aragón (CEICA). Se reclutaron 15 (7 mujeres y 8 varones) sujetos que se presentaron en la consulta de podología y biomecánica de forma voluntaria y se obtuvo el consentimiento informado de todos ellos. Se excluyeron aquellos sujetos menores de edad, que no estuviesen en el rango de índice de masa corporal normopeso (entre 18,5 y 24,5), que hubieran sufrido una lesión o una intervención en la extremidad inferior que pudiera alterar el patrón de la carrera del sujeto.

Se utilizó una cinta de correr BH (modelo i.F2W Dual). Para simular la carrera se utilizó una velocidad de $10 \mathrm{~km} / \mathrm{h}$ con una inclinación nula, es decir, 0 grados de inclinación respecto al suelo.

Se utilizó un smartphone iPhone $8^{\circledR}$ para grabar las mediciones, que integra una cámara de $12 \mathrm{Mpx}$, capaz de grabar vídeos a cámara lenta en 1080p a 120 fps. El dispositivo estuvo localizado, en todas las mediciones realizadas a los sujetos, a $1 \mathrm{~m}$ del eje central de la cinta de correr y a una altura de $62,5 \mathrm{cms}$ del suelo, gracias a un trípode. De esta forma aparecieron las extremidades inferiores por completo.
Se siguió el mismo protocolo de grabación de forma rigurosa para todos los participantes. Los sujetos realizaron la prueba con unas mallas completamente negras y descalzos. Para medir el ángulo de flexo-extensión de la rodilla y del tobillo se colocó un diodo emisor de luz $(L E D)$ de color verde en las principales referencias óseas de la extremidad inferior: trocánter mayor, cóndilo externo del fémur, maléolo externo y apófisis estiloides del quinto metatarsiano (Figura 1). Los sujetos corrieron en la cinta a una velocidad de 10 $\mathrm{km} / \mathrm{h}$, obteniendo tres grabaciones del ciclo de la carrera de cada participante en la primera sesión. Se realizó una segunda sesión de grabaciones a los 7 días del primer registro para volver a grabar siguiendo el mismo protocolo otras tres grabaciones del ciclo de la carrera de cada participante.

$\mathrm{Se}$ procesaron todas las grabaciones obtenidas mediante el software Younext Direct Motion Capture ${ }^{\circledR}$ para obtener los datos cuantitativos. El algoritmo utilizado por $O p e n C V$ proporciona la posición espacial del pixel del color seleccionado en toda la filmación, por lo que se conocen sus coordenadas en el eje horizontal (eje X) y en el eje vertical (eje Y) de todo el movimiento que se pretende analizar. Se combina de una forma automática la información procedente de 3 píxeles distantes entre sí para obtener un ángulo relativo a los segmentos definidos (fémur-tibia y tibia-pie). El punto central siempre será el fulcro sobre el que rotarán los puntos más distales, obteniendo de esta forma el ángulo en todos los frames.

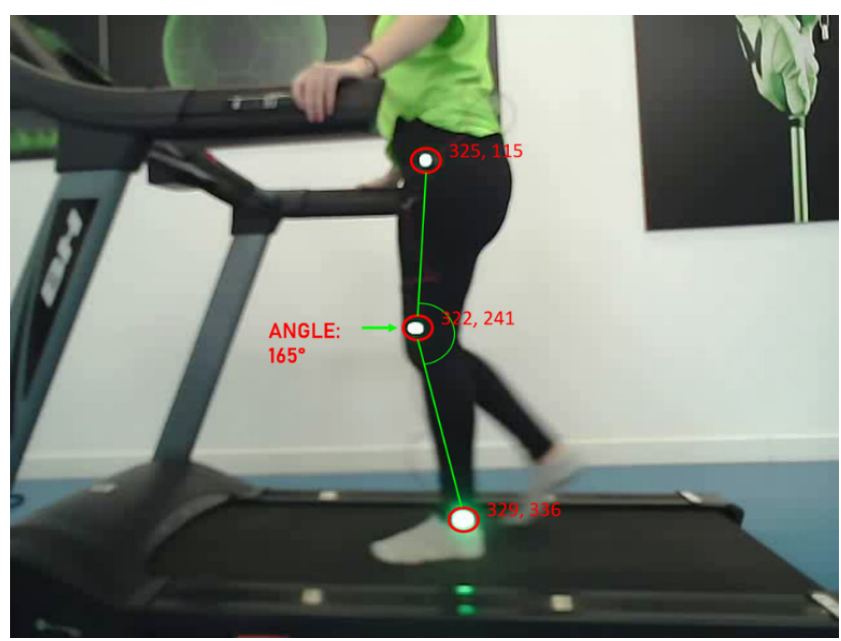

Figura 1. Medición de la flexo-extensión de la rodilla con Younext Direct Motion Capture $\mathbb{R}$ 
Se realizó el análisis estadístico mediante SPSS 20.0 para Windows (SPSS Ibérica, Madrid, España). Para los datos antropométricos de la muestra se calcularon la media y la desviación típica con un intervalo de confianza del 95\%. Para la fiabilidad intrasesión e inter-sesión se calculó el coeficiente de correlación intraclase (ICC). Para interpretar los resultados, se utilizó como referencia la clasificación propuesta por Landis y Koch(24): 0,20 o menos, leve concordancia; 0,21 a 0,40 concordancia discreta; 0,41 a 0,60 concordancia moderada; 0,61 a 0,80 concordancia sustancial y 0,81 o más concordancia casi perfecta. Se calculó, a partir de la media y la desviación típica, el coeficiente de variación $(\mathrm{CV})$ para la fiabilidad intra-sesión. Un CV alto $(>5 \%)$ indicará una gran heterogeneidad de los parámetros. Por último, el error estándar de la medida (EEM) se calculó para medir el rango de error de la flexo-extensión de la rodilla y del tobillo durante la carrera. Se calculó entre las sesiones, por medio del ICC y la desviación típica.

\section{Resultados}

Debido a los criterios de inclusión y pérdidas de seguimiento, 8 sujetos ( 5 varones y 3 mujeres) con una edad media de $25,5 \pm 1,07$ y un IMC medio de $22,0 \pm 1,92 \mathrm{~kg} / \mathrm{m}^{2}$ completaron el estudio. El diagrama de flujo detallado del estudio se describe en la Figura 2. Respecto a las características socio-demográficas de la muestra, éstas se detallan en la Tabla 1.

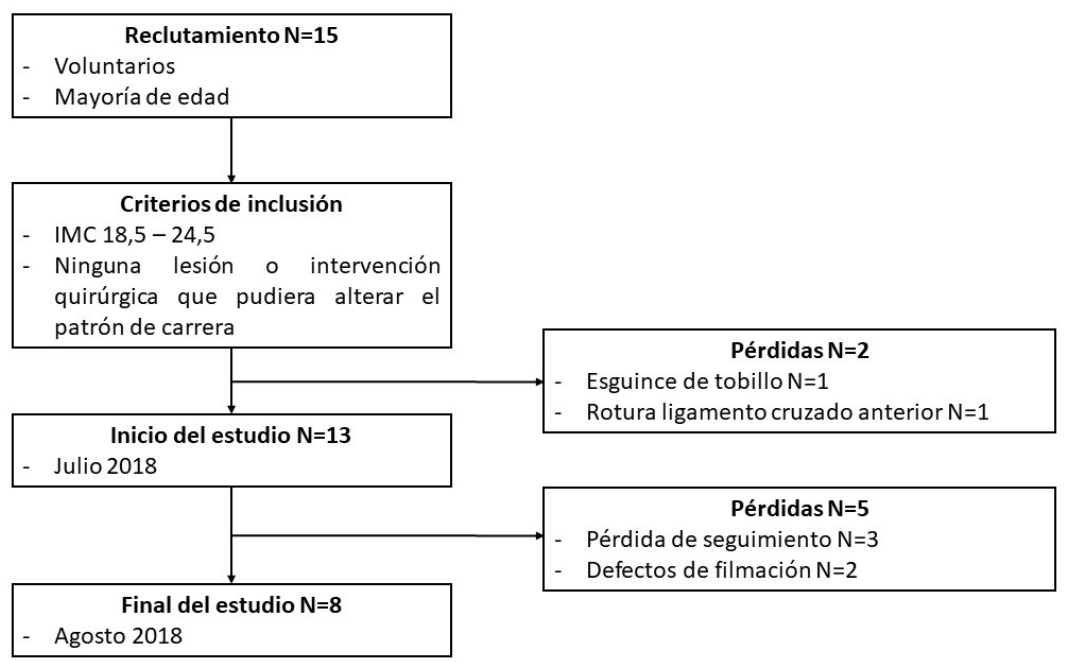

Figura 2. Diagrama de flujo

Tabla 1. Características socio-demográficas de la muestra

\begin{tabular}{|c|c|}
\hline VARIABLE & Media \pm Dt $\mathbf{( 9 5 \%}$ IC) \\
\hline Edad & $25,5 \pm 1,07(24,6-26,4)$ \\
\hline Altura (cm) & $175,1 \pm 8,63(167,9-182,3)$ \\
\hline Peso (kg) & $67,9 \pm 10,6(59,0-76,7)$ \\
\hline IMC (kg/m2) & $22,0 \pm 1,92(20,4-23,6)$ \\
\hline
\end{tabular}

Abreviaciones: Dt: Desviación típica; IC: Intervalo de confianza; IMC: Índice de masa corporal. Se consideró un valor de $\mathrm{p}<0,05$ como estadísticamente significativo. 
La fiabilidad intra-sesión de la primera sesión se presenta en la Tabla 2. Como se puede observar, se obtuvo una concordancia casi perfecta $(\mathrm{ICC}>0,81)$ para la medición del ángulo de la rodilla $(0,981)$ y del tobillo $(0,996)$ con valores EEM muy bajos. Se obtuvo una ligera mejor correlación en las mediciones del tobillo. Sin embargo, observando los coeficientes de variación, éstos son superiores al $5 \%$ en las mediciones de tobillo, obteniendo $6,54 \%$. Por el contrario, para la medición angular de la rodilla, el coeficiente de variación es inferior al $5 \%$, obteniendo $3,17 \%$ para la primera sesión.

Tabla 2. Fiabilidad intra-sesión de la sesión 1

\begin{tabular}{|c|c|c|c|c|}
\hline Variable & Media \pm Dt & $\begin{array}{c}\text { ICC } \\
(\mathbf{9 5 \%} \text { IC) }\end{array}$ & CV (\%) & EEM \\
\hline $\begin{array}{c}\text { Flexo-extensión } \\
\text { Rodilla }\end{array}$ & $140,87 \pm 4,46$ & $\begin{array}{c}0,981 \\
(0,939-0,996)\end{array}$ & 3,17 & 0,61 \\
\hline Flexo-extensión Tobillo & $122,34 \pm 8,00$ & $\begin{array}{c}0,996 \\
(0,988-0,999)\end{array}$ & 6,54 & 0,51 \\
\hline
\end{tabular}

Abreviaciones: Dt: Desviación típica; ICC: Coeficiente de correlación intraclase [3, k]; IC: Intervalo de confianza; CV: Coeficiente de variación; EEM: Error estándar de la medida.

La fiabilidad intra-sesión de la segunda sesión se presenta en la Tabla 3. Al igual que en la primera sesión, esta vez se obtuvo de nuevo una concordancia casi perfecta (ICC > $0,81)$ tanto para las mediciones de la rodilla como para el tobillo. De nuevo, se obtuvo una ligera mejor concordancia para el tobillo
$(0,997)$ que para la rodilla $(0,994)$ con unos valores EEM muy bajos. Observando los coeficientes de variación se observó una mayor variabilidad en las mediciones del tobillo, que volvieron a ser superiores al 5\% $(6,77 \%)$ y una menor variabilidad, inferior al $5 \%$, para las mediciones de la rodilla $(3,46 \%)$.

Tabla 3. Fiabilidad intra-sesión de la sesión 2

\begin{tabular}{|c|c|c|c|c|}
\hline VARIABLE & Media \pm Dt & $\begin{array}{c}\text { ICC } \\
(\mathbf{9 5 \%} \text { IC) }\end{array}$ & CV (\%) & EEM \\
\hline $\begin{array}{c}\text { Flexo-extensión } \\
\text { Rodilla }\end{array}$ & $141,10 \pm 4,89$ & $\begin{array}{c}0,994 \\
(0,982-0,999)\end{array}$ & 3,46 & 0,38 \\
\hline Flexo-extensión Tobillo & $122,65 \pm 8,30$ & $\begin{array}{c}0,997 \\
(0,990-0,999)\end{array}$ & 6,77 & 0,45 \\
\hline
\end{tabular}

Abreviaciones: Dt: Desviación típica; ICC: Coeficiente de correlación intraclase [3, k]; IC: Intervalo de confianza; CV: Coeficiente de variación; EEM: Error estándar de la medida.

La repetibillidad inter-sesión se presenta en la Tabla 4. Se obtuvo una concordancia casi perfecta para la medición angular de la rodilla $(\mathrm{ICC}=0,975)$ y del tobillo (ICC $=0,989)$ con unos valores EEM muy bajos. De nuevo, la correlación obtenida para el tobillo fue ligeramente más elevada que la de la rodilla, sin embargo, respecto a los coeficientes de variación, se observó que éste era superior en la medición angular del tobillo $(6,65 \%)$ que en la medición angular de la rodilla $(3,31 \%)$. 
Tabla 4. Fiabilidad inter-sesión

\begin{tabular}{|c|c|c|c|c|}
\hline VARIABLE & Media \pm Dt & $\begin{array}{c}\text { ICC } \\
(\mathbf{9 5 \%} \text { IC) }\end{array}$ & CV (\%) & EEM \\
\hline $\begin{array}{c}\text { Flexo-extensión } \\
\text { Rodilla }\end{array}$ & $140,98 \pm 4,67$ & $\begin{array}{c}0,975 \\
(0,877-0,995)\end{array}$ & 3,31 & 0,74 \\
\hline $\begin{array}{c}\text { Flexo-extensión } \\
\text { Tobillo }\end{array}$ & $122,5 \pm 8,15$ & $\begin{array}{c}0,989 \\
(0,950-0,998)\end{array}$ & 6,65 & 0,85 \\
\hline
\end{tabular}

Abreviaciones: Dt: Desviación típica; ICC: Coeficiente de correlación intraclase [3, k]; IC: Intervalo de confianza; CV: Coeficiente de variación; EEM: Error estándar de la medida.

\section{Discusión}

El objetivo de este estudio fue determinar la fiabilidad y la repetibilidad de la cinemática angular de la rodilla y del tobillo de la carrera en el plano sagital en población sana utilizando Younext Direct Motion Capture ${ }^{\circledR}$.

En este estudio se examinó una muestra adulta sana y se demostró que el software Younext Direct Motion Capture ${ }^{\circledR}$ proporciona mediciones fiables para la medición angular de la rodilla y del tobillo, con una concordancia casi perfecta. Sin embargo, para una fiabilidad absoluta se debe tener en cuenta otros indicadores, como son el coeficiente de variación y el error estándar de la medida. En términos de variabilidad, únicamente las mediciones de rodilla tuvieron valores por debajo al 5\%. El error estándar de la medida, también conocido como error típico de la medida, es una expresión cuantitativa del rango de errores que puede ocurrir cuando un sujeto repite ciertas mediciones. En este estudio se observó unos valores de EEM muy bajos, lo que indica una fiabilidad absoluta sólida.

Según Schurr et.al, se han observado relaciones importantes entre el análisis 2D y $3 \mathrm{D}$ en todas las articulaciones en el plano sagital(22). El alto coste de los sistemas 3D, la fragilidad del sensor y la complejidad de funcionamiento hacen que estos sistemas se utilicen principalmente para la investigación. Younext Direct Motion Capture ${ }^{\circledR}$ es un sistema de análisis de movimiento $2 \mathrm{D}$ con una sencilla aplicación que no requiere una gran cualificación para su uso.

Las principales limitaciones del estudio estuvieron relacionadas con el tamaño de la muestra. Al tratarse de un estudio piloto, se calculó una muestra pequeña. El hecho de haber obtenido unos valores de ICC casi perfectos con una muestra tan pequeña invita a conocer si se obtendrían los mismos resultados con una muestra mayor. A esta pequeña muestra se añadieron algunas pérdidas por defectos de filmación. Al igual que otros autores, algunos vídeos fueron imposibles de analizar por la baja calidad y por ello se excluyeron del análisis. La calidad de las grabaciones depende de una iluminación suficiente al grabar a alta velocidad movimientos como la carrera. Por lo tanto, hay que destacar que, antes de utilizar técnicas de análisis de movimiento en $2 \mathrm{D}$ en la práctica clínica, es importante evaluar el entorno clínico y la configuración general(25).

Sería interesante determinar en un futuro la fiabilidad de Younext Direct Motion Capture ${ }^{\circledR}$ en la cadera, ya que se ha demostrado que los sistemas de análisis 2D son fiables a la hora de cuantificar la cinemática de la cadera en corredores sanos(25). Con las principales articulaciones de la extremidad inferior analizadas, se podría estudiar la efectividad del sistema para medir la evolución de posibles intervenciones tras alguna lesión en la extremidad inferior. Este programa podría servir como una herramienta rápida y eficaz para la evaluación en entornos clínicos o deportivos, lo que podría contribuir a la identificación temprana de la patología en la carrera. Aunque una medición puede ser fiable en un sujeto joven y saludable, lo mismo puede no ser cierto para una persona con un patrón de marcha anormal, por lo que se necesita un estudio adicional en una población de pacientes diferente.

En conclusión, este estudio ha demostrado que el software Younext Direct Motion Capture $^{\circledR}$ es fiable para analizar la cinemática angular de la rodilla y del tobillo de la carrera en población adulta sana. 


\section{Bibliografía}

1. DiPietro L. Physical activity in aging: changes in patterns and their relationship to health and function. J Gerontol A Biol Sci Med Sci [Internet]. 2001 Oct [cited 2018 Jun 16];56 Spec No 2:13-22. Available from: http://www.ncbi.nlm.nih.gov/pubmed/11730234

2. Warburton DER, Nicol CW, Bredin SSD. Health benefits of physical activity: the evidence. Can Med Assoc J [Internet]. 2006 Mar 14 [cited 2018 Jun 16];174(6):801-9. Available from: http://www.ncbi. nlm.nih.gov/pubmed/16534088

3. Pisinger C, Toft U, Aadahl M, Glümer C, Jørgensen T. The relationship between lifestyle and self-reported health in a general population: the Inter99 study. Prev Med (Baltim) [Internet]. 2009 Nov [cited 2018 Jun 16];49(5):418-23. Available from: http://linkinghub.elsevier.com/retrieve/pii/S0091743509004137

4. Boone-Heinonen J, Evenson KR, Taber DR, Gordon-Larsen P. Walking for prevention of cardiovascular disease in men and women: a systematic review of observational studies. Obes Rev [Internet]. 2009 Mar [cited 2018 Jun 16];10(2):204-17. Available from: http://www.ncbi.nlm.nih.gov/pubmed/19207874

5. Hamer M, Chida Y. Walking and primary prevention: a meta-analysis of prospective cohort studies. Br J Sports Med [Internet]. 2008 Apr 1 [cited 2018 Jun 16];42(4):238-43. Available from: http://www.ncbi. nlm.nih.gov/pubmed/18048441

6. Murtagh EM, Murphy MH, Boone-Heinonen J. Walking: the first steps in cardiovascular disease prevention. Curr Opin Cardiol [Internet]. 2010 Sep [cited 2018 Jun 16];22(5):490-6. Available from: http://www.ncbi.nlm.nih.gov/pubmed/20625280

7. Schnohr P, Marott JL, Lange P, Jensen GB. Longevity in Male and Female Joggers: The Copenhagen City Heart Study. Am J Epidemiol [Internet]. 2013 Apr 1 [cited 2018 Jun 16];177(7):683-9. Available from: http://www.ncbi.nlm.nih.gov/pubmed/23449779

8. Koplan JP, Rothenberg RB, Jones EL. The natural history of exercise: a 10-yr follow-up of a cohort of runners. Med Sci Sports Exerc [Internet]. 1995 Aug [cited 2018 Jun 16];27(8):1180-4. Available from: http://www.ncbi.nlm.nih.gov/pubmed/7476063

9. Nielsen RO, Buist I, Sørensen H, Lind M, Rasmussen S. Training errors and running related injuries: a systematic review. Int J Sports Phys Ther [Internet]. 2012 Feb [cited 2018 Jun 16];7(1):58-75. Available from: http://www.ncbi.nlm.nih.gov/pubmed/22389869

10. Krebs DE, Edelstein JE, Fishman S. Reliability of observational kinematic gait analysis. Phys Ther [Internet]. $1985 \mathrm{Jul}$ [cited 2018 Jun 17];65(7):1027-33. Available from: http://www.ncbi.nlm.nih.gov/ pubmed/3892553

11. Speciali DS, de Oliveira EM, dos Santos NM, Pereira FV, Fracini AC, Fukuda TY, et al. Use of the Gait Deviation Index and spatiotemporal variables for the assessment of dual task interference paradigm. J Bodyw Mov Ther [Internet]. 2013 Jan [cited 2018 Jun 17];17(1):19-27. Available from: http:// linkinghub.elsevier.com/retrieve/pii/S1360859212001106

12. Bilney B, Morris M, Webster K. Concurrent related validity of the GAITRite walkway system for quantification of the spatial and temporal parameters of gait. Gait Posture [Internet]. $2003 \mathrm{Feb}$ [cited 2018 Jun 17];17(1):68-74. Available from: http://www.ncbi.nlm.nih.gov/pubmed/12535728

13. Valentini FA, Granger B, Hennebelle DS, Eythrib N, Robain G. Repeatability and variability of baropodometric and spatio-temporal gait parameters--results in healthy subjects and in stroke patients. Neurophysiol Clin [Internet]. 2011 Oct [cited 2018 Jun 17];41(4):181-9. Available from: http:// linkinghub.elsevier.com/retrieve/pii/S0987705311000669

14. Maffiuletti NA, Gorelick M, Kramers-de Quervain I, Bizzini M, Munzinger JP, Tomasetti S, et al. Concurrent validity and intrasession reliability of the IDEEA accelerometry system for the quantification of spatiotemporal gait parameters. Gait Posture [Internet]. 2008 Jan [cited 2018 Jun 17];27(1):160-3. Available from: http://linkinghub.elsevier.com/retrieve/pii/S0966636207000306

15. Lieberman DE, Venkadesan M, Werbel WA, Daoud AI, D'Andrea S, Davis IS, et al. Foot strike patterns and collision forces in habitually barefoot versus shod runners. Nature [Internet]. 2010 Jan 28 [cited 2018 Jun 17];463(7280):531-5. Available from: http://www.nature.com/articles/nature08723

16. Daoud AI, Geissler GJ, Wang F, Saretsky J, Daoud YA, Lieberman DE. Foot strike and injury rates in endurance runners: a retrospective study. Med Sci Sports Exerc [Internet]. 2012 Jul [cited 2018 Jun 17];44(7):1325-34. Available from: https://insights.ovid.com/crossref?an=00005768-201207000-00017

17. Bertelsen ML, Jensen JF, Nielsen MH, Nielsen RO, Rasmussen S. Footstrike patterns among novice runners wearing a conventional, neutral running shoe. Gait Posture [Internet]. 2013 Jun [cited 2018 Jun 17];38(2):354-6. Available from: http://linkinghub.elsevier.com/retrieve/pii/S0966636212004481

18. Nolan L, Patritti BL, Simpson KJ. A biomechanical analysis of the long-jump technique of elite female amputee athletes. Med Sci Sports Exerc [Internet]. 2006 Oct [cited 2018 Jun 17];38(10):1829-35. Available from: https://insights.ovid.com/crossref?an=00005768-200610000-00019

19. Kivi DMR, Maraj BK V, Gervais P. A kinematic analysis of high-speed treadmill sprinting over a range 
of velocities. Med Sci Sports Exerc [Internet]. 2002 Apr [cited 2018 Jun 17];34(4):662-6. Available from: http://www.ncbi.nlm.nih.gov/pubmed/11932576

20. Munro A, Herrington L, Carolan M. Reliability of 2-dimensional video assessment of frontal-plane dynamic knee valgus during common athletic screening tasks. J Sport Rehabil [Internet]. 2012 Feb [cited 2018 Jun 20];21(1):7-11. Available from: http://www.ncbi.nlm.nih.gov/pubmed/22104115

21. Wille CM, Lenhart RL, Wang S, Thelen DG, Heiderscheit BC. Ability of Sagittal Kinematic Variables to Estimate Ground Reaction Forces and Joint Kinetics in Running. J Orthop Sport Phys Ther [Internet]. 2014 Oct [cited 2018 Jun 17];44(10):825-30. Available from: http://www.ncbi.nlm.nih.gov/ pubmed/25156183

22. Schurr SA, Marshall AN, Resch JE, Saliba SA. TWO-DIMENSIONAL VIDEO ANALYSIS IS COMPARABLE TO 3D MOTION CAPTURE IN LOWER EXTREMITY MOVEMENT ASSESSMENT. Int J Sports Phys Ther [Internet]. 2017 Apr [cited 2018 Jun 17];12(2):163-72. Available from: http://www.ncbi.nlm.nih.gov/pubmed/28515970

23. Nidhi. Image Processing and Object Detection. Int J Appl Res [Internet]. 2015 Aug 1 [cited 2018 Jun 18];1(9):396-9. Available from: http://www.allresearchjournal.com/ archives $/$ ?year $=2015 \& \mathrm{vol}=1 \&$ issue $=9 \&$ part $=$ G\&ArticleId $=615$

24. Landis JR, Koch GG. The Measurement of Observer Agreement for Categorical Data. Biometrics [Internet]. 1977 Mar [cited 2018 Jun 18];33(1):159. Available from: https://www.jstor.org/ stable/2529310?origin $=$ crossref

25. Damsted C, Nielsen RO, Larsen LH. Reliability of video-based quantification of the knee- and hip angle at foot strike during running. Int J Sports Phys Ther [Internet]. 2015 Apr [cited 2018 Aug 30];10(2):14754. Available from: http://www.ncbi.nlm.nih.gov/pubmed/25883863 